Canad. Math. Bull. Vol. 18 (2), 1975

\title{
NOTE ON A SUBRING OF $C^{*}(X)^{(1)}$
}

\author{
BY \\ CHOO ENG-UNG
}

Throughout, topological spaces are assumed to be completely regular. $C(X)$ (resp. $C^{*}(X)$ ) will denote the ring of all (resp. all bounded) continuous real-valued functions. $\beta X$ will denote the Stone-Cech compactification of $X$. In [2], Nel and Riorden defined $C^{\neq}(X)$ to be the set of all $f \in C(X)$ such that $M(f)$ is real in the residue class ring $C(X) / M$ for every maximal ideal $M$ in $C(X) . C^{\neq}(X)$ is a subalgebra as well as a sublattice of $C^{*}(X)$. Some equivalent topological and algebraic characterizations of $C^{\neq}(X)$ are given. The main aim of this paper is to prove that $X$ is pseudocompact iff $C^{\neq}(X)=C(X)$ iff $C^{\neq}(X)$ determines the topology of $X$ and is uniformly closed. All notations and background information are referred to [1].

LEMMA. If $\left\{x_{n}: n \in N\right\}$ is a subset of a zero set $Z$ of $X$ such that $\lim _{n \rightarrow \infty} f\left(x_{n}\right)=r$ and $r \notin f[Z]$, then $\left\{x_{n}: n \in N\right\}$ contains a C-embedded copy of $N$.

Proof. Let $Z=Z(g)$ and $h=1 /\left((f-r)^{2}+g^{2}\right)$. Then $h \in C(X)$. Since $h$ is unbounded on $\left\{x_{n}: n \in N\right\}$, hence $\left\{x_{n}: n \in N\right\}$ contains a $C$-embeddable copy of $N[1,1.20]$.

The equivalence of (1) and (3) in the following theorem was given in [2].

THEOREM. For a function $f \in C(X)$ the following are equivalent.

(1) $f \in C^{\neq}(X)$

(2) $f \in C^{*}(X)$ and $p \in \mathrm{Cl}_{\beta X}\left[Z\left(f-f^{\beta}(p)\right)\right]$ for every $p \in \beta X$.

(3) $f \in C^{*}(X)$ and $f[D]$ is finite for every $C$-embedded copy $D$ of $N$.

(4) $f \in C^{*}(X)$ and $f[Z]$ is closed for every zero set $Z$ of $X$.

Proof. We show $(1) \rightarrow(2) \rightarrow(3) \rightarrow(4) \rightarrow(1)$.

$(1) \rightarrow(2)$. Let $f \in C^{\neq}(X)$. Then $f \in C^{*}(X)[1,5.7]$. For any $p \in \beta X$, since $M^{P}(f)$ is real, hence $f-r \in M^{P}$ for some $r \in R$. It follows that $p \in \mathrm{Cl}_{\beta X}[Z(f-r)]$ and $f^{\beta}(p)=r$.

$(2) \rightarrow(3)$. Let $p \in \mathrm{Cl}_{\beta X} D$. Then $p \in \mathrm{Cl}_{\beta X}\left[Z\left(f-f^{\beta}(p)\right)\right] \cap \mathrm{Cl}_{\beta X} D$. If $Z\left(f-f^{\beta}(p)\right) \cap$ $D=\varnothing$, then $\mathrm{Cl}_{\beta X} Z\left(f-f^{\beta}(p)\right) \cap \mathrm{Cl}_{\beta X} D=\varnothing[1,1.18]$. Thus $Z\left(f-f^{\beta}(p)\right) \cap D \neq \varnothing$ and hence $f^{\beta}(p) \in f[D]$. Hence $f[D]=f^{\beta}\left[\mathrm{Cl}_{\beta X} D\right]$ is closed. It follows that $f[D]$ is finite.

$(3) \rightarrow(4)$. Assume that $f[Z]$ is not closed for some zero set $Z$. Let $r \in$ $\mathrm{Cl}_{R}[f[Z]]-f[Z]$. Choose $x_{n} \in Z(n \in N)$ such that $\lim _{n \rightarrow \infty} f\left(x_{n}\right)=r$. Then, by the

Received by the editors June 6, 1973 and, in revised form, September 17, 1973.

(1) This paper is a part of the author's Ph.D. thesis at the University of British Columbia written under the supervision of J. V. Whittaker. 
lemma, $\left\{x_{n}: n \in N\right\}$ contains a $C$-embedded copy $D$ of $N$. It is obvious that $f[D]$ is not finite.

$(4) \rightarrow(1)$. Let $M$ be any maximal ideal of $C(X)$. Then $M=M^{P}$ for some $p \in \beta X$ $[1,7.3]$. Assume that $f-f^{\beta}(p) \notin M^{P}$. Then there exists a zero set $Z$ such that $p \in \mathrm{Cl}_{\beta X} Z$ and $Z \cap Z\left(f-f^{\beta}(p)\right)=\varnothing$. Since $f[Z]$ is closed, hence $f^{\beta}(p) \in f[Z]$. This is a contradiction. Therefore, $f-f^{\beta}(\dot{p}) \in M^{P}$ and hence $M(f)$ is real.

There is an interesting algebraic characterization of $C^{\neq}(X)$ which depends only on the maximal ideals in $C(X)$ and $C^{*}(X)$.

THEOREM. $C^{\neq}(X)$ is the largest subring of $C^{*}(X)$ satisfying:

(1) $C^{\neq}(X)$ contains all the constant functions, and

(2) $M^{p} \cap C^{f}(X)=M^{* p} \cap C^{\neq}(X)$ for every $p \in \beta X$.

Proof. Suppose $G$ is a subring of $C^{*}(X)$ satisfying conditions (1) and (2). Let $g \in G$. For every $p \in \beta X$, the function $g-g^{\beta}(p) \in M^{* p} \cap G$. By condition (2), $g-g^{\beta}(p) \in M^{p} \cap G$. Thus, $p \in \mathrm{Cl}_{\beta X}\left[Z\left(g-g^{\beta}(p)\right)\right]$. Therefore, $g \in C^{\neq}(X)$. Hence $G \subseteq C^{\neq}(X)$. It remains to show that $C^{\neq}(X)$ satisfies the condition (2). For every $p \in \beta X$, it is obvious that $M^{p} \cap C^{\neq}(X) \subseteq M^{* p} \cap C^{\neq}(X)$. Let $f \in M^{* p} \cap C^{\neq}(X)$. Then $f^{\beta}(p)=0$ and $p \in \mathrm{Cl}_{\beta X}\left[Z\left(f-f^{\beta}(p)\right)\right]=\mathrm{Cl}_{\beta X}[Z(f)]$. Hence, $f \in M^{p}$. Consequently, $M^{p} \cap C^{\neq}(X)=M^{* p} \cap C^{\neq}(X)$ for every $p \in \beta X$.

We are now ready to prove the main theorem.

THEOREM The following are equivalent.

(1) $X$ is a pseudocompact space.

(2) $C^{f}(X)=C(X)$.

(3) $C^{\neq}(X)$ determines the topology of $X$ and $C^{\neq}(X)$ is uniformly closed.

Proof. We show (1) $\rightarrow(2) \rightarrow(3) \rightarrow(1)$.

$(1) \rightarrow(2)$. Since $C(X)=C^{*}(X)$, hence, $M^{p}=M^{* p}$ for every $p \in \beta X$.

By previous theorem, it follows that $C^{\neq}(X)=C(X)$.

$(2) \rightarrow(3)$. It is obvious.

(3) $\rightarrow(1)$. Suppose $X$ is not pseudocompact. Then $X$ has a $C$-embedded copy $D=\left\{x_{n}: n \in N\right\}$. Let $\left\{O_{n}: n \in N\right\}$ be a countable collection of disjoint open sets where $x_{n} \in O_{n}$ for every $n \in N$. For every $n \in N$, there exists $f_{n} \in C^{\neq}(X)$ such that

(i) $f_{n}\left(x_{n}\right)=1 / 2^{n}$.

(ii) $Z\left(f_{n}\right)$ contains $X-O_{n}$. and

(iii) $O \leq f_{n} \leq 1 / 2^{n}$.

Let $g=\sum_{n=1}^{\infty} f_{n}$. Since $C^{\neq}(X)$ is uniformly closed, hence $g \in C^{\neq}(X)$. But $g[D]$ is not closed. This is a contradiction. Consequently, $X$ is a pseudocompact space.

REMARK. It can be proved that if $X$ is a locally compact space, then $C^{\neq}(X)$ determines the topology of $X$. It is difficult to find a space $X$ where $C^{\neq}(X)$ does not determine the topology of $X$. 


\section{REFERENCES}

1. L. Gillman and M. Jerison, Rings of Continuous Functions, Van Nostrand, N.Y., 1960.

2. L. D. Nel and D. Riordan, Note on a Subalgebra of $C(X)$, Cand. Math. Bull. Vol. 15 (4), 1972.

UNIVERSiTy of SCIENCE of MALAYSIA,

Penang, Malaysia 